\title{
Conversational Agency in Augmentative and Alternative Communication
}

\author{
Stephanie Valencia, Amy Pavel, Jared Santa Maria, Seunga (Gloria) Yu, \\ Jeffrey P. Bigham, Henny Admoni \\ Human-Computer Interaction Institute and Robotics Institute \\ Carnegie Mellon University \\ \{svalenci,apavel,jsantama,seungay,jbigham,hadmoni\}@ andrew.cmu.edu
}

\begin{abstract}
Augmented communicators (ACs) use augmentative and alternative communication (AAC) technologies to speak. Prior work in AAC research has looked to improve efficiency and expressivity of AAC via device improvements and user training. However, ACs also face constraints in communication beyond their device and individual abilities such as when they can speak, what they can say, and who they can address. In this work, we recast and broaden this prior work using conversational agency as a new frame to study AC communication. We investigate $\mathrm{AC}$ conversational agency with a study examining different conversational tasks between four triads of expert ACs, their close conversation partners (paid aide or parent), and a third party (experimenter). We define metrics to analyze AAC conversational agency quantitatively and qualitatively. We conclude with implications for future research to enable ACs to easily exercise conversational agency.
\end{abstract}

\section{Author Keywords}

AAC; accessibility; agency; cerebral palsy; conversation.

\section{INTRODUCTION}

Agency is not a fixed property, but rather manifests within structure [11] and constraints [14] that challenge its expression. People with severe speech and physical impairments may communicate using AAC devices such as eye tracking-based speech generators or pointing-based picture boards [4]. Such computer-mediated communication brings unique constraints to both the AAC device user and their conversation partners. For example, when a communication partner knows that it takes someone using an AAC device more time to respond, the communication partner may ask yes/no questions instead of open-ended ones, limiting the AC's possible responses [18].

As a first step towards making communication possible and efficient, designers and researchers of AAC have focused on improving device throughput, for example via text prediction [34], and vocabulary sorting [2, 21], and improving

Permission to make digital or hard copies of part or all of this work for personal or classroom use is granted without fee provided that copies are not made or distributed for profit or commercial advantage and that copies bear this notice and the full citation on the first page. Copyrights for third-party components of this work must be honored For all other uses, contact the Owner/Author(s).

CHI '20, April 25-30, 2020, Honolulu, HI, USA.

(C) 2020 Copyright is held by the owner/author(s).

ACM ISBN 978-1-4503-6708-0/20/04.

http://dx.doi.org/10.1145/3313831.3376376

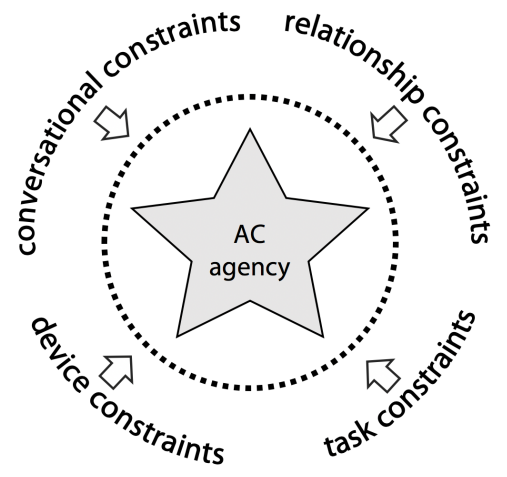

Figure 1. Conversational constraints (e.g., who can speak, who can be addressed), the task at hand, the relationship with other speakers, and the device being used can all impact the expression of an augmented communicator's (AC) agency in conversation.

expressivity, with awareness displays [32] and customizable voices [12, 27, 29]. Research from communication science has focused on increasing communicative competence of ACs [23, 25]. Other researchers have recognized social factors including the role of communication partners in how an AC communicates [13]. Recent AAC research has started to take a critical view of how AAC technology mediates communication [20], but there is still no model of how constraints specific to AAC impact the expression of agency in AAC in-person interactions. Prior work has not yet determined how different types of conversational partners impact an AC's agency.

In this work, we use conversational agency as a frame to study AAC user's experiences in conversation. We specifically consider Gibson's definition from sociology [14], which explains that individuals can exercise agency when conversational constraints loosen enough for them to contribute to the conversation. These conversational constraints include when a person can speak, whom they can address, what can be said, and what can be expected from others by way of cooperation. In addition to conversational constraints, we also consider constraints that are particularly relevant in the case of AAC communication and task-oriented dialogue (Figure 1): relationship with a communication partner (e.g., years knowing each other, comfort, previous communication experience), device properties (e.g., throughput, input type, vocabulary, feedback type), and task constraints (e.g., communication channel available, output type, information owner). We are interested in understanding how ACs exercise conversational agency in the 


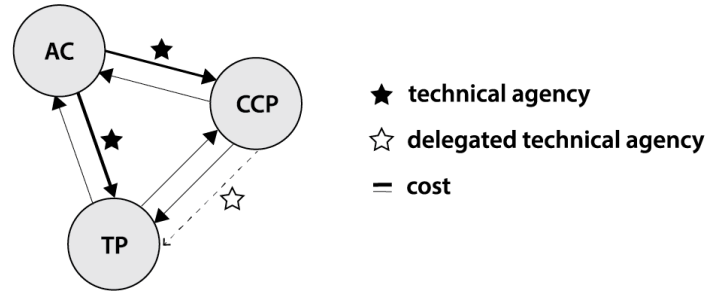

Figure 2. Conversational, task, relationship and device constraints can change the pathways for communication (arrows) between AC, CCPs and a third party (TP) and the effort, or cost, required to communicate along the pathways (arrow thickness). For example, time-sensitive conversational constraints (e.g., replying fast while it is still relevant) can encourage communication along the lowest cost paths (lower width arrows) encouraging communication via their CCP rather than direct $\mathrm{AC}$ to TP communication. When this delegated technical agency enables the realization of goals for ACs we call it Colloquial Agency.

face of unique constraints such as the device they use, their relationship with the people they are talking with, the task at hand and the general conversational constraints explained by Gibson (Figure 1).

To address this, we carried out an observational study with ACs and their close conversational partners (CCPs). CCPs such as parents and paid aides, who have caregiving roles, often support ACs by facilitating conversations with others [5]. We consider scenarios in which there is an unfamiliar third party (TP) to study how CCPs facilitate the conversation between the AC and the third party, and how this impacts the ACs' agency. Our findings show that CCPs support ACs' agency by supporting communication with third parties. CCPs also increase the amount of information exchanged on behalf of the $\mathrm{AC}$ at a lower cost to the $\mathrm{AC}$ through explanations and by breaking down complex questions into yes/no questions. But, such explanations and narrow questions can lead to a reduced set of $\mathrm{AC}$ responses, and missed opportunities for $\mathrm{AC}$ to participate. In this paper, we contribute an empirical understanding of agency in AAC and conclude with ideas on how future designs can consider CCP participation while facilitating ACs' to exercise their agency in conversation.

\section{BACKGROUND AND RELATED WORK}

\section{Conversational Agency, a Matter of Constraints}

Agency is expressed through actions that further an individual's objective in the face of outside structure or constraints [7, $11,15,30]$. Gibson, in his work on Conversational Agency, distinguishes two types of agency. Technical agency, is exercised when individuals contribute to the conversation (Figure 2). As individuals express technical agency by participating they can then advance their objectives in conversation, which he identifies as colloquial agency [14]. According to Gibson, an individual's technical agency can be promoted or reduced by four core conversational constraints (Table 1). We define these constraints and describe how they matter for AAC conversations.

The one-speaker constraint specifies that only one speaker can speak at a time. When one speaker cues that they are almost done, another actor can preemptively start speaking to secure the floor. The one-speaker constraint implies that turn

\begin{tabular}{lll}
\hline Constraints & Governs & Contributing factors \\
\hline One speaker & Current speaker & Time to start speaking \\
Participation shift & Next speaker & Addressing next speaker (e.g. by gaze, name, topic) \\
Relevance & Topic of contribution & Time spent speaking without pause \\
Ritual & Type of contribution & Previous speech act (e.g. question) \\
\hline
\end{tabular}

Table 1. We summarize the four conversational constraints that impact agency according to Gibson, what governs the constraint, and factors that contribute to the constraint application.

changes are a valuable resource for securing the opportunity for technical agency. When using an AAC device, ACs are at a disadvantage. Non-augmented speakers can secure a turn faster by speaking directly, while ACs have to operate their device to do this. Prior work has highlighted that ACs have challenges with social cuing or indications of turn-taking, as their speakers fail to recognize when ACs want to contribute to conversation [22].

The relevance constraint states that a new contribution to a conversation will be interpreted as a response to the most recent prior contribution. Thus, the relevance of a particular response decreases as the conversation proceeds. Together with the one-speaker constraint, the relevance constraint suggests that a very short window of time is available for an AC to make a relevant contribution to the conversation.

The participation shift constraint states that the target of a prior remark has the greatest odds of speaking next, but any participant may speak next. The prior target's advantage builds as speaking provides other participants more opportunities for engagement. A known problem in AAC conversation is that ACs are often not addressed directly by third parties, instead third parties talk to them via their CCP. CCPs are recommended to help third parties engage directly with the AC by explaining that they can communicate directly [6].

The ritual constraint suggests conversation participants seek to maintain a positive social standing by showing considerateness to the speaker, assisting them with repair, and eliciting sympathy to them. Either through experience, exposure or training, CCPs may have specific ways in which they support their ACs' communication [6]. We expect to see these rituals, such as waiting for a response or assisting in a particular way, to play a supportive role for ACs to exercise their agency in conversation.

\section{Agency in $\mathrm{HCl}$}

Prior work in HCI has generally defined agency as a sense of control over the external environment and one's body [26]. Shneiderman's Eight Golden Rules of Interface Design [31] encourages interfaces that "support an internal locus of control," inspiring work evaluating perceived agency for interactions. In particular, such work considers implicit "sense of agency" measures (e.g., perceived time of action and outcome) to study the impact of interface feedback [10]. In this work, we examine matters of agency beyond sense of control during an individual experience with an interface, and into agency as a social experience, impacted by context and others.

\section{In-person AAC Interactions}

Prior observational [16, 17, 20], survey [13], and interview $[1,21,22]$ studies have investigated AAC conversations 
among augmented communicators and close conversational partners identifying challenges to CCP-assisted conversation. This work motivates our study of CCPs' impact on an AC's agency under conversational, task, device, and relationship constraints.

Recent work in AAC has pointed to the importance of studying how conversational partners can impact ACs' agency. Ibrahim et al. observed children using their AAC devices to communicate with adults and their peers. They found that adults erroneously corrected the children's speech when they assumed children's competence using the device was low [20]. Adults also recounted a child's prior communicative act by accessing and reading out a previously constructed utterance with the AAC device without the child's consent. In our study, we consider CCP collaboration in the case of expert, adult, AAC users who face different challenges to their agency in conversation. Goodwin, who studied AAC use with people with aphasia, notes that agency for ACs occurs within a social context where the meaning of communication must be formed cooperatively $[16,17]$. We build upon this work by studying the participation of CCPs in forming meaning from AC's communication. In particular, we investigate how such support increases or hinders ACs' agency across different tasks.

Communication scientists studying AAC in-person conversations have used methods such as conversational micro-analysis, examining interactions at the utterance level to understand participation in conversations $[8,9,19,24,29]$. We use similar micro-analysis methods to quantify participation by identifying specific occurring communicative functions. We add to this body of work by analyzing participation across a set of different tasks to specifically investigate the relationship between CCP participation and ACs' expression of agency.

Prior studies on conversation-partner-assisted AAC communication further inform our inclusion of task, relationship, and device constraints in framing our study of ACs' agency. Fiannaca et al. surveyed ACs and their caregivers about caregiverassisted communication, finding that the caregiver assistance can be uncomfortable when conversing with groups, and frustrating when the caregiver guesses incorrectly [13]. To better understand how such assistance may support or hinder AC participation, we observe CCP-AC pairs in conversation with a third party (TP), and in completing goal-oriented tasks. ACs may communicate more effectively with close companions in familiar settings than with strangers in unfamiliar settings [1, 22] suggesting relationship may impact cost of communication (Figure 1). Adults who adopted AAC devices later in life (due to ALS) reported becoming passive speakers who consider the cost of communicating when deciding to participate [22]. This suggests that agency may be also limited by the requirements needed to operate a device, such as energy and effort.

\section{STUDYING AGENCY, APPROACH AND STUDY SETUP}

Our approach is to study the participation of close conversational partners (CCPs) to understand how to facilitate augmented communicators (ACs) to exercise their agency. In doing so, we address the following research questions:

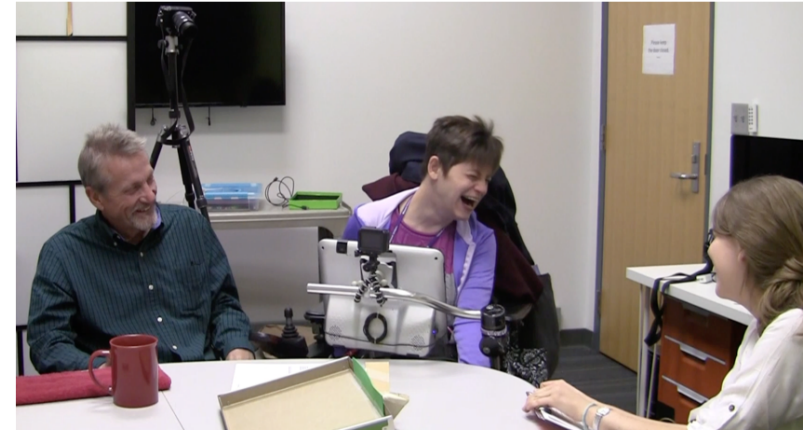

Figure 3. Study setup. The CCP (left), the AC (middle) and the experimenter(right) sit at a round table. A scene camera captures this image, a camera behind the AC captures activity on the AAC device, and a camera mounted on the AC's wheelchair captures AC's facial expressions.

RQ1: How do conversational, relationship, device and task constraints impact CCPs' participation?

RQ2: How does CCPs' participation impact ACs' agency?

RQ3: When does technical agency impact colloquial agency?

\section{Tasks}

We use three tasks to investigate how factors such as potential to speak (e.g., who holds the knowledge in the conversation, and who are the active participants) and goals (e.g., an answer to a question, versus a creative decision) impact ACs' technical and colloquial agency when working with a CCP. We include the participation of a third-party (TP), who is an unfamiliar conversation partner to the AC to analyze differences due to the relationship constraint (Figure 3). The TP (experimenter) has experience communicating with ACs but not with these particular participants. According to Blackstone's Circles of Communication Partners [5], the TP holds a relationship similar to partners in the fourth circle (e.g., workers who communicate with ACs as part of their profession) and the fifth circle (e.g., people ACs have not met before).

\section{Interview task}

We conducted experimenter-led semi-structured interviews with each $\mathrm{AC}$ and $\mathrm{CCP}$ pair in order to 1) investigate $\mathrm{AC}$ technical agency in a structured conversation assisted by a $\mathrm{CCP}$, and 2) gather background information about the AAC device and the AC-CCP pair. The experimenter asked all questions directly to the AC. Questions were about the device and the ACs' experience with it. These are topics in which the AC and CCP could potentially both know the answers, so they shared the same potential to respond (Figure 4, far left). The experimenter addresses all questions directly to the $\mathrm{AC}$, to control the participation shift constraint and evaluate if this helped the AC secure turns and participate more. We expected the interview to encourage open conversation to answer RQ1.

\section{Map task}

Inspired by prior work $[18,19]$ that used maps to examine how ACs and their partners achieve common ground, we investigated how CCP-AC partnerships differ from TP-AC partnerships when working together to complete a map. For this task the $\mathrm{AC}$ has access to a map displaying a route between 

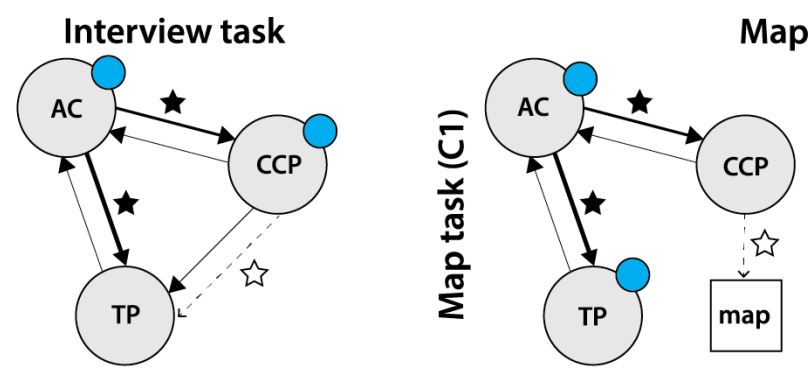

Map task
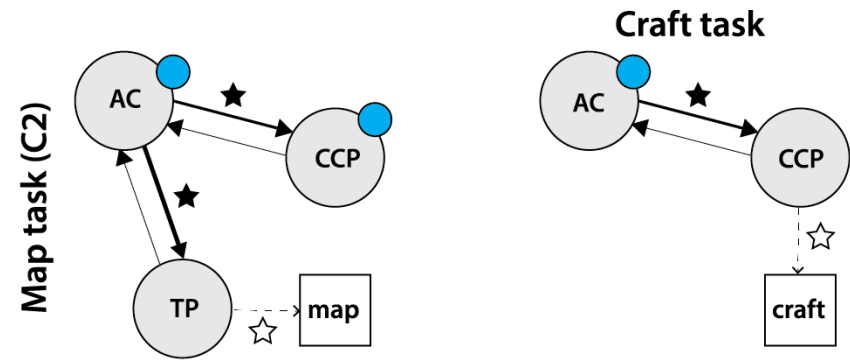

Figure 4. The interview task mirrors open conversation except that the third party only directly addresses the AC (missing arrow between the third party and CCP) and only the $\mathrm{AC}$ and $\mathrm{CCP}$ share knowledge to answer the interview questions (small blue dots). In the first map task condition (C1), the $\mathrm{AC}$ and the third party can see the correct route (small blue dots) and the AC must communicate to the CCP what to draw on the map. In the second map condition, the AC and CCP share the correct route and must tell the third party what to draw on the map. In the craft task, the AC generates ideas for their craft which is largely constructed by the CСP (the third party does not participate).

landmarks and must direct another person (either CCP or TP) to draw the given route on their blank map.

In condition one (map C1), the TP acts as an assistive partner who can also see the map and the CCP is the follower who has the blank map (Figure 4, second from left). In map condition two (map C2), the CCP is the assistive partner and the TP is the follower (Figure 4, second from right). We use the map task to answer RQ1 and RQ2 and also explore RQ3 to understand what type of interactions contributed to the AC being able to successfully communicate the map's route (technical agency) and their partner drawing it correctly on the map (colloquial agency). Higginbotham et al. $[18,19]$ found that CCP often set rules or strategies to solve the map task in an efficient way, so we hypothesize the setting of rules or strategies as a potential action from CCPs that can impact ACs' expression of agency. We also expect to see different interaction patterns when there is mainly AC-to-CCP communication (condition 1 ), as opposed to AC-to-TP communication (condition 2).

\section{Craft task}

To further explore RQ3, we designed a craft task in which the $\mathrm{AC}$ and CCP collaborated to design an image collage (Figure 4, far right). The TP did not participate in this task. Similar to the map task, we designed a craft task to explore how AC and CCP collaboration supports the translation of ideas expressed by the AC (technical agency) to design decisions on the final collage artifact (colloquial agency). The craft task involves choices based on preference rather than a single correct route as in the map task. In this activity, the AC holds the knowledge of their creative choices, while the CCP can physically explore the materials available and physically creates the final artifact through delegated agency.

\section{Operationalizing Agency}

We used quantitative video coding analysis to develop both overall and task-specific metrics for technical agency, colloquial agency and CCP participation to address our research questions. We considered the amount of conversational contributions made by CCPs and ACs and classified them with specific communicative function types as in $[8,24]$. To address RQ3, we also examined the resulting artifacts of the map and craft task to evaluate how participation (technical agency) impacted the map or craft (colloquial agency).
To measure conversational contributions we first annotated the start and end of each participation event in the videos (e.g., the CCP speaks to give an explanation, the AC gestures to confirm). Then, each participation event was labelled with an ID (e.g., CCP, AC, third party), the form of the interaction(e.g., speaking, gesturing, vocalizing), and the type of communicative function (e.g., giving an explanation, confirming information). We selected communicative functions labels specific to our research questions (see supplemental material for full guide) and only labelled actions, gestures and vocalization that served a communicative function as in [8]. Two coders watched all of the videos. We determined inter-rater reliability from a sample of $25 \%$ of video data coded independently by two coders. We sampled each 0.5 second, and achieved a Cohen's Kappa of 0.87 overall communicative functions labels for all participation events. Videos were transcribed and time-marked to capture sequences of utterances and turns.

\section{AC technical agency}

We measured occurrences of AC's technical agency as individual contributions to the conversation through speech, gestures, and vocalizations. Through all tasks, we consider the length and number of conversational turns taken in each modality. In the interview, we separately considered technical agency (whether an AC gives an explanation or a yes/no answer to a question), and delegated technical agency (CCP answers the question with AC's permission). We do this by identifying specific AC communicative functions such as: requesting help (RH) or time (RT), giving an explanation (E), granting/denying permission (GP/DP), and confirming/denying information the $\mathrm{CCP}$ has said, other than a permission request (COI/NOI). In the map tasks, we considered the number of strategies explicitly stated by the CCP and the AC to solve the task. In the craft task, we considered the total number of ideas proposed by the $\mathrm{CCP}$ and the $\mathrm{AC}$.

\section{AC colloquial agency}

We examined the resulting maps routes and collage elements as a proxy for colloquial agency (achieving individual objectives). For the map task, we considered the number of accurate and inaccurate landmarks on the final map. For the craft task, we considered each element in the collage, examining who proposed the element (e.g., CCP or AC) and who agreed to the idea of including the element (e.g., none, CCP, or AC). 


\begin{tabular}{|c|c|c|c|c|c|c|}
\hline ID & Age & Gender & $\begin{array}{l}\text { Years with AAC; } \\
\text { with Current Device }\end{array}$ & Device WPM & AAC Device Set Up & Partner \\
\hline $\mathrm{AC} 1$ & 23 & M & $19 ; 10$ & 2.65 & $\begin{array}{l}\text { Tobii Dynavox Maestro; head-controlled switch and scan- } \\
\text { ning access; phrase-level voice output }\end{array}$ & Mother (CCP1) \\
\hline $\mathrm{AC} 2$ & 38 & $\mathrm{~F}$ & $32 ; 1.5$ & 8.94 & $\begin{array}{l}\text { Tobii Dynavox i-12; wheelchair joystick for direct access; } \\
\text { phrase-level voice output }\end{array}$ & Father (CCP2) \\
\hline AC3 & 50 & M & $35 ; 1.5$ & 7.03 & $\begin{array}{l}\text { Accent } 1000 \text {; direct access using head tracker; utterance- } \\
\text { level voice output }\end{array}$ & Paid aide for 0.7 yrs (CCP3) \\
\hline $\mathrm{AC} 4$ & 50 & $\mathrm{~F}$ & $33 ; 23$ & 22.24 & $\begin{array}{l}\text { DeltaTalker; head-mounted pointer for direct access; utter- } \\
\text { ance level voice output }\end{array}$ & Paid aide for 4 yrs (CCP4) \\
\hline
\end{tabular}

Table 2. Our eight study participants, four augmented communicators (ACs) and their corresponding close conversation partners (CCPs). Using our transcripts, we calculated the throughput or total words spoken over minutes typing (WPM) for each AC.

\section{CCP participation}

For all tasks, we related CCP participation to the AC's participation (technical agency) by calculating similar metrics to those calculated for the AC: number of conversational turns, amount of speaking time per task, number of strategies stated in the map task and number of ideas contributed during craft task. In addition to this, we annotated specific types of CCP participation by communicative function: asking permission to explain $(\mathrm{P})$, giving an explanation $(\mathrm{E})$, lending assistance $(\mathrm{A})$ such as conversational repair and physical assistance through actions, and asking clarifying questions (QC) to the AC.

\section{PARTICIPANTS AND DATA COLLECTION}

We recruited 8 participants: 4 expert augmented communicators (ACs) with cerebral palsy and their corresponding close conversational partners (CCPs) (Table 2). We call ACs expert AAC users as they have been using AAC devices for more than 10 years, on a daily basis. AC participants used either phrase-level voice output, speaking a phrase or word once it was completely typed, or utterance-level voice output, for which ACs spoke utterances as they formed a word (e.g., "H-er-Hero"). In addition to their device, AC participants also used nonspeech vocalizations (e.g., grunting) and facial gestures (e.g., blinks, smiles and eye-contact) to communicate. All $\mathrm{ACs}$ had closed fists and some used hand restrains (AC1 and $\mathrm{AC} 4)$. AC2 and AC3 used arm gestures to communicate. We collected audio and video recordings from three video cameras to capture all tasks: 1) a camera placed at a distance to capture the entire scene including all participants, 2) a camera placed behind the AC to capture the AAC device's screen, and 3) a small GoPro camera in front of the AC (either on the AC's wheelchair mount or on the table in front of the AC) to capture facial expressions.

\section{Procedure}

An experimenter (the first author) first conducted semistructured interviews with participant pairs, directing all questions to the $\mathrm{AC}$ with the $\mathrm{CCP}$ present. Interviews took between 35 and 55 minutes to complete including rest breaks. We then gave participants a limited amount of time ( $\mu=12$ minutes) to complete each of the two map task conditions. We counterbalanced the order of the conditions between participants. For each pair, we used two randomly selected maps from the HRCR map corpus ${ }^{1}$. We edited these maps to include large

\footnotetext{
${ }^{1}$ http://groups.inf.ed.ac.uk/maptask/maptasknxt.html
}

font and minimal graphics, and provided them to the participants on paper. After the map task, participants spent 12-18 minutes completing the craft task. We provided participant pairs with materials to create a collage craft that included construction paper, magazines, writing utensils, stickers, scissors, tape and glue. Pairs could make a collage either: 1) for a loved one to give as a gift, or 2) about a place they wanted to visit on vacation. We invited each pair to take home their collage.

At the end of the study we asked each pair a series of debrief questions together, and then a different set of questions to each AC and CCP individually (without the other present). With both participants present, we asked about likes, dislikes and challenges encountered during study tasks. To the AC and the CCP individually, we asked about strategies and challenges experienced while communicating with their partner and with others outside of the study.

\section{Study Limitations}

Having these interactions in a lab setting with obvious video recording might have limited the types of behaviors ACs and CCPs used. Nevertheless, we observed a range of communication strategies and meaningful differences across tasks on the way participants behaved. Our study investigates specific partner configurations as described in Figure 4 but does not encompass all possible conversation scenarios. There are many other types of AC-CCP relationships that may impact agency and need to be investigated (e.g., "friend-tendants" or colleagues). Similarly, there are other types of third parties that need to be studied. The third party (experimenter) is a person familiar with AAC that did not know the participants previously. This resembles the scenario of an AC who meets a professional for the first time and is accompanied by their CCP. However, we do not examine interactions with completely novice third parties.

\section{RESULTS: CONSTRAINTS AND PARTICIPATION}

Our first research question asked: How do conversational, relationship, device, and task constraints impact CCP participation? Conversational constraints regulated CCP participation by balancing turns and enabling topic dominance through long contributions. CCPs who were parents to the AC participated much more than paid aides across all tasks. ACs using devices with lower WPM experienced more CCP participation. The task goals and the access to the information needed to complete the task also impacted CCP participation. 


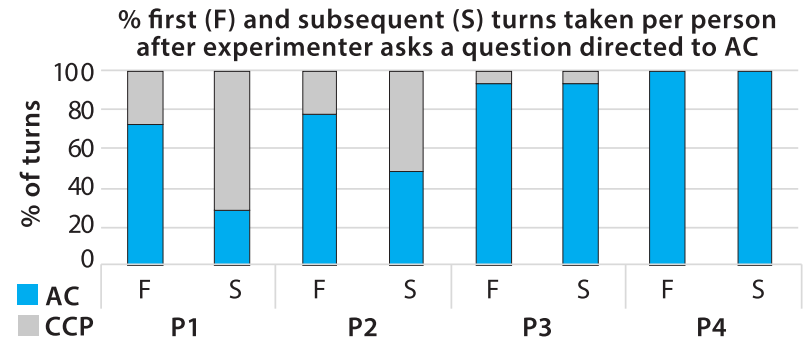

Figure 5. Overall, ACs contributed a higher percentage of turns immediately after they were addressed by the third party (F). CCPs in pairs 1 and 2 , contributed a higher percentage of the time in subsequent turns (S).

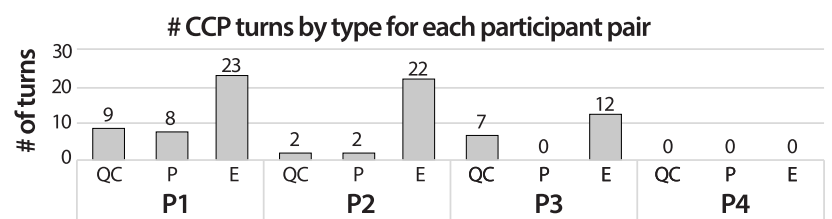

Figure 6. When CCPs took a turn during the interview, they most often gave explanations (E). CCPs also asked questions for confirmation by the $\mathrm{AC}(\mathrm{QC})$ and asked permission before providing explanations $(\mathrm{P})$.

Effect of Conversational Constraints on Participation CCPs participated less when the third party directly addressed the AC in the interview task and participated more in subsequent turns (participation shift constraint). CCPs also balanced their own explanations by asking clarifying questions or asking for permission before answering or expanding on a question on behalf of the AC (ritual constraint) engaging in a collaborative sharing of information. Long turns by the CCPs, often during AC typing, impacted ACs' opportunity to make relevant contributions (relevance constraint). CCP participation lowered when the $\mathrm{AC}$ took more turns and longer turns and used utterance-level voice output that allowed the $\mathrm{AC}$ to hold the floor while typing (one-speaker constraint).

\section{Participation shift constraint}

The participation shift constraint states that an individual has more opportunities to contribute when addressed directly by the current speaker. Conforming to this constraint, the AC spoke more often relative to the $\mathrm{CCP}$ when directly addressed by the third party (Figure 5). When CCPs participated in subsequent turns after the AC took the first turn, CCPs expanded on ACs' responses by (1) giving an explanation of the ACs' response to provide context (with or without permission), and (2) asking the AC a follow up question to achieve clarity. During such explanations, CCPs often expanded on ACs' yes/no responses to the third party:

TP: Do you use your device for social media?

AC2: No

CCP2: Yes and no ... We just don't do it at the moment...

The CCP most often responded first to the third party when the AC struggled to answer the question. For example, after one of the interview questions CCP2 asked for permission to respond on behalf of $\mathrm{AC} 2$ after noticing she was stuck on a page on her device and was taking a long time to answer the question. As the duration of the interview increased, the participation shift constraint loosened, participation shifted to

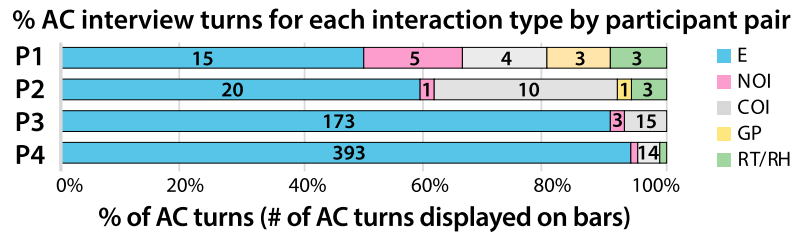

Figure 7. When ACs took a turn during the interview, they most often gave explanations (E). Other turns were spent confirming (COI) or denying (NOI) information given by the CCP, granting permission (GP) to CCPs to provide explanation, requesting help (RH) and requesting time (RT). ACs in P1 and P2 explained in less than $60 \%$ of turns, while $\mathrm{ACs}$ in $\mathrm{P3}$ and $\mathrm{P4}$ explained in more than $90 \%$ of turns.

and from all directions (CCP to $\mathrm{TP}, \mathrm{CCP}$ to $\mathrm{AC}, \mathrm{AC}$ to $\mathrm{CCP}$ ), and the CCP spoke more often. The only exception was CCP4 who only contributed to clarify AC4's device pronunciation.

\section{Ritual constraint}

The ritual constraint suggests that participants regulate the conversation to maintain a positive social standing (e.g., by involving a new speaker, answering a speaker's question, or filling silence). CCPs upheld the ritual constraint by providing direct explanations (E) which served to answer questions and fill silences. They also upheld the ritual constraint by asking permission before giving an explanation $(\mathrm{P})$ and by asking clarifying questions (QC), which served to involve the AC (Figure 6). ACs reciprocated clarifying questions with a simple confirmation or denial of the information if they were the next to speak:

TP: Is there any other device that you use for communication in general or for computer access?

AC1: Yes.

CCP1: To use the device as a computer, is that what you are thinking?

AC1: [Blinks once to say "no."]

CCP1: ... Or are you thinking about the iPad? The one I am thinking of ...

AC1: Low-tech.

CCP1: Oh there is low-tech. Oh my gosh, thank you!

When CCPs asked for permission, they did not necessarily wait for permission to be granted by the AC. For instance, AC1 only granted permission on 4 of the 8 occasions when CCP1 asked (Figures 6 and 7). CCPs also provided a portion of their explanations while the AC was typing (CCP1, CCP2, and CCP3 gave $50 \%, 47 \%$ and $12 \%$ of their explanations during typing respectively; $\mathrm{CCP} 4$ did not give any explanation).

\section{Relevance constraint}

As CCP participation increased in subsequent turns, conversation topics moved quickly and the time window ACs had to respond shrank, tightening the relevance constraint. In consequence, when ACs finished typing something they wanted to say, they had to choose to either erase it, or to say it even if this required making an off-topic comment. For example, on two occasions, $\mathrm{AC} 1$ typed a relevant answer but then erased it as the conversation continued and the comment lost relevance, all while CCP1 was speaking. The first time AC1 erased his contribution, the CCP was giving a long explanation (1.8 minutes). The second time, $\mathrm{AC} 1$ erased a contribution after the 


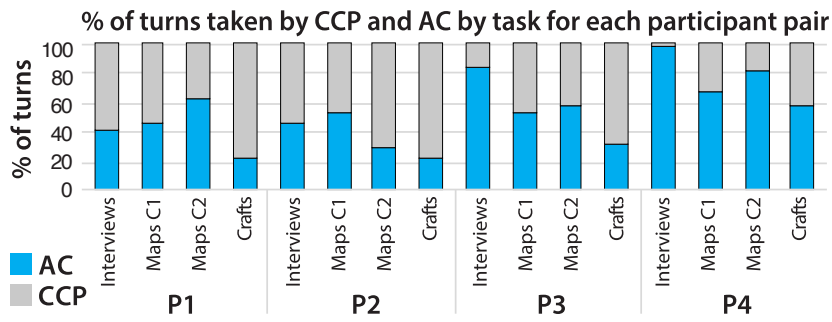

Figure 8. Percentage of turns taken by the $\mathrm{AC}$ and $\mathrm{CCP}$ per task for each participant pair.

third party asked a second question and CCP1 replied to it with an explanation that took about a minute:

TP: For how long have you been using the Series 5?

CCP1: Yeah for how long... Do you know?

AC1: [Gestures no by blinking once, begins typing.]

CCP1: I am going to say like ten years ... he is actually been using a device for 19 years ... [continues explaining for a long time]

TP: Do you use your device every day?

AC1: [Erases message typed, unintelligible.] Can you hold on a minute, please?

TP: Yes.

AC1: Every day.

On two other occasions, $\mathrm{AC} 1$ and $\mathrm{AC} 2$ contributed off-topic comments to the conversation. In one instance, CCP1 was trying to remember the name of old hardware that $\mathrm{AC} 1 \mathrm{had}$ used. CCP1 eventually changed the topic. After nearly two minutes, $\mathrm{AC} 1$ spoke the hardware's name. CCP1 was taken by surprise. The second off-topic comment also occurred after a long explanation by $\mathrm{CCP} 2$, that had caused the topic to move on. In contrast, CCP3 and CCP4 spent less time explaining and offered explanations less often, and neither $\mathrm{AC} 3$ or $\mathrm{AC} 4$ experienced off-topic or erased comments.

\section{One speaker constraint}

Talking over ACs' speech utterances as they are being formed is a violation of the one speaker constraint. Participants with utterance-level speech (AC3, AC4) engaged in more openended participation, providing more explanations during the interview (Figure 7) than participants with phrase-level speech $(\mathrm{AC} 1, \mathrm{AC} 2)$. AC3 and $\mathrm{AC} 4$ were able to participate more (Figure 8 ) and were less likely to be interrupted.

\section{Effect of Relationship Constraints on Participation}

CCPs who are parents to the $\mathrm{AC}$ and therefore lifelong CCPs (CCP1, CCP2) participated more often across all tasks (Figure 8). In the interview, parents took speaking turns to provide explanations and ask for permission before explaining (Figure 6), while paid aides (CCP3, CCP4) participated primarily to clarify ACs' contributions and speech. In the map task, lifelong partners and aids participated relatively equally. In the crafts task, lifelong partners participated more and contributed more craft ideas (Table 3 ), while paid aides took comparatively fewer turns relative to ACs.

Across all tasks, ACs interacting with lifelong CCPs also used gestures and vocalizations to communicate more often than ACs interacting with paid aides (Figure 9). In one instance,

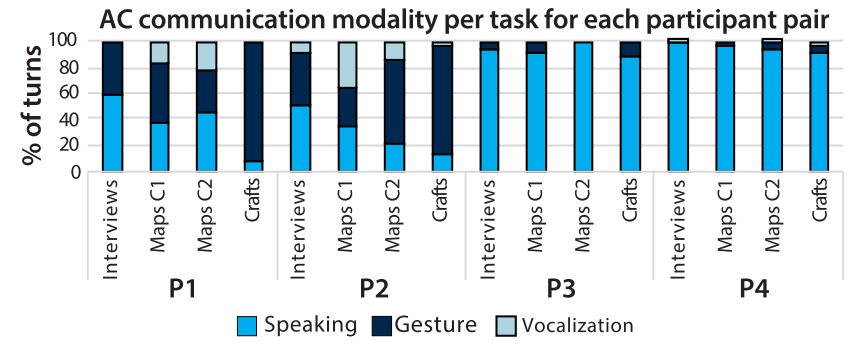

Figure 9. Modes of communication used by AC per task

during an explanation about word pronunciation by CCP1, $\mathrm{AC} 1$ communicated with a glance to the CCP. In response, CCP1 immediately chuckled, saying "alright, I will stop talking now". In another instance, when the third party asked AC2 an interview question, AC2 extended her hands to her father, CCP2, who immediately responded by holding her hands and answering the question. Unlike paid aides, lifelong CCPs occasionally interacted directly with the AAC device by directly operating the device to complete a word (CCP1) or moving the device to one side to allow the $\mathrm{AC}$ more room to color and choose materials in the craft task (CCP2).

\section{Effect of Task Constraints on Participation}

Access to information needed to complete each task, the task's goals, and the communication partners' roles all impacted CCP participation. All participants completed all tasks except for pair 2, who did not complete either map condition by decision of $\mathrm{AC} 2$, who found the task very challenging.

\section{Access to task-relevant information}

CCPs contributed substantive explanations and ideas when they had access to information relevant for the task. In the interview task, CCP1 and CCP2 (lifelong CCPs) contributed more explanations based on their knowledge of the AC's experiences (Figure 6). In the craft task, CCP1 and CCP2 knew the recipients of their collages, and as a result suggested more ideas than CCP3 and CCP4 (Table 3).

In contrast, when $\mathrm{ACs}$ uniquely held the information required to complete the task, like in the map $\mathrm{C} 1$ and craft tasks (the route to the map and their creative ideas, respectively), all CCPs, except CCP2, participated more often by actively retrieving information from ACs (Figure 8). In particular, CCPs asked yes/no, short answer, and follow-up questions to try and retrieve information quickly (e.g., "what is the first letter of the next location?", "what should we do?" and "how about this picture of the beach?").

\section{Task goals}

Under the pressure of the time-constrained, goal-oriented map tasks, CCPs participated less often than in the more timeflexible, open-ended craft task (Figure 8). In the map task, CCPs proposed many strategies and quickly settled on a strategy once they found a successful approach (CCP1, CCP2, CCP3, and CCP4 proposed 7, 4, 3, and 9 strategies respectively across both conditions). ACs participated by stating directions in the map task, but ACs infrequently suggested strategies (only AC2 and AC4 proposed one strategy each over both conditions). Although CCP suggested strategies intended to make the task easier ("you don't have to give me the whole 
word ...just give me a couple letters"), the strategies limited the ways that ACs could carry out the task. For instance, CCP1 suggested AC1 type the first letter of each landmark and CCP1 would type the rest. But when AC1 typed the letter "f," CCP1 mistakenly completed the word "finish" twice, even though AC1 tried erasing and spelling another word, "Flag ship," which was the last landmark on the route. The strategy set by CCP1 prevailed and it was challenging for AC1 to correct it. In the collaborative craft task, CCPs took more turns relative to ACs to suggest multiple ideas for the crafts, allowing ACs more options before settling on a solution.

\section{Communication Partners' roles}

CCPs provided assistance more frequently when performing tasks that required primarily AC-to-TP communication compared to when performing tasks that required primarily AC-toCCP communication (Figure 4). For the interview and map $\mathrm{C} 2$ task, which mainly required AC-to-TP communication, the CCP frequently assisted and repaired communication between the $\mathrm{AC}$ and the third party by clarifying device errors and pronunciations (in the interview) and groundings (in the map). During the interview, CCPs provided 18, 17, 11, and 3 clarifications respectively. During map C2, CCPs grounded the conversation by questioning the TP to check if they understood the AC's communication (e.g., "do you have that landmark?", "yeah, he blinked twice, that is a no," "she is typing"). In the craft and map C1 tasks, which required AC-to-CCP communication, ACs used gestures and vocalizations more often than in tasks that required AC-to-TP communication (Figure 9).

\section{Effect of Device Constraints on Participation}

Properties of the AAC device (e.g., throughput and input type) impacted $\mathrm{AC}$ and $\mathrm{CCP}$ participation. $\mathrm{AC} 1, \mathrm{AC} 2$, and $\mathrm{AC} 3$, who experienced lower device throughput (Table 2), displayed high ratios of CCP-to-AC participation (Figure 8) compared to AC4 who experienced the highest device throughput. While $\mathrm{CCP} 1, \mathrm{CCP} 2$, and CCP3 gave explanations in the interview (Figure 6), CCP4 participated only 3 times to clarify device pronunciation. AC4 also managed the conversation (e.g. " "oh, let me think about that," "did I answer your question?") and elaborated more on her answers compared to other ACs. On the other hand, CCP1 facilitated abbreviated communication by $\mathrm{AC} 1$, who had the lowest WPM, by requesting that the AC type individual letters instead of full words in the map task.

\section{RESULTS: PARTICIPATION AND TECHNICAL AGENCY}

Our second research question asked: How does CCPs' participation impact ACs' technical agency? We identified that CCP participation: 1) supported AC contributions by creating multi-modal communication channels, 2) increased information exchanged at a lower cost via delegated agency, but also 3 ) reduced AC contributions through missed opportunities.

\section{Supporting Independent AC's Communication}

CCPs facilitated communication with third parties by knowing how to interpret their $\mathrm{AC}$ partner's unique communication characteristics. For example, CCPs assisted in repairing misunderstandings by clarifying AAC device pronunciation, informed the third party about the AC's status when there was a device error, and let the third party know what $\mathrm{AC}$ gestures or nonverbal behaviors meant. CCP attributes (e.g., previous experience, relationship) enabled this process. Thus, the presence of a close conversation partner opens new communication paths for the $\mathrm{AC}$ and extends their communication possibilities beyond the AAC device. Physically, CCPs also enable otherwise challenging tasks (e.g., bringing maps closer for ACs to see, or moving crafting materials closer for ACs to draw with) to support independent AC contributions. Outside of the study, CCPs can encourage third parties to elicit AC contributions, as mentioned during the debrief interviews:

"One of the things I do to help with that is by turning my body towards him, so that they are not relating to me but they are relating to him. I am helping orient the conversation towards him." - CCP1

\section{Increasing Information Exchanged}

CCPs can increase information being shared in a conversation with a third party by expanding on information previously provided by the $\mathrm{AC}$ with permission from the $\mathrm{AC}$ (delegated technical agency) or by directly answering questions and confirming the information with the $\mathrm{AC}$ afterwards:

"I would ask him, would you like me to explain that more?
... but if he would like to say it himself he could continue
but I will usually will help him when I know there is more
to something." - CCPI

The latter strategy facilitates confirmation or correction of the information by the AC, but it may leave less room for independent $\mathrm{AC}$ explanations as there is no time allowed for the AC to directly answer or frame their response as they desire. ACs confirm that outside of the study, CCPs often anticipate what the $\mathrm{AC}$ will say by guessing (AC1, AC3, AC4), or by looking directly at the device (AC4). But at the same time, one AC reported that such guessing is "useful 50\% of the time" and AC4 reported that while she does not prefer over-the-shoulder reading it can be occasionally useful:

"[At] church, it's often times noisy so in that case I don't mind if a person looks on my display. ... I would rather be understood than them hearing me." - AC4

AC4 mentioned that whether she liked other people guessing varied depending on how she was feeling:

"It varies. If I am on edge I do not appreciate people anticipating my words. I can and I want to articulate myself and my way. But that isn't me very much. I am pretty easy going." - AC4

When ACs were asked for ideas of what CCPs could do instead of guessing, AC1 explained: "I am not sure - because they can't read my mind. But if it is something I usually need, it is useful." AC3 said he preferred guessing when using headspelling, in which his CCP would guess as he spelled letters using his head.

\section{Creating Missed Opportunities}

Though CCPs aim to increase information exchange and lower the cost of expression for ACs, their actions can actually reduce ACs' technical agency by creating "missed opportunities" to contribute. For example, ACs occasionally erased what 


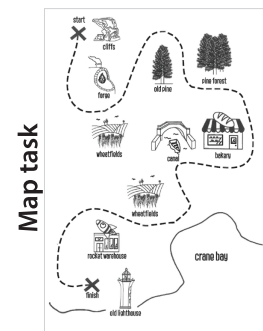

Guide map
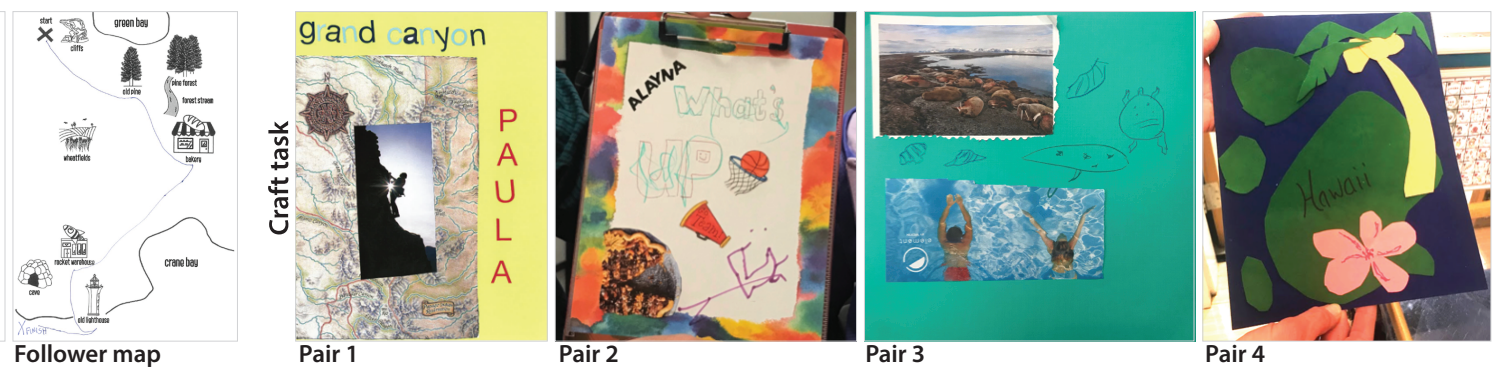

Figure 10. Example materials for the map and craft task. For the map task, we show an example guide map with route information (visible by AC) and follower map (visible by CCP or third party) that shows only the starting point, requiring the follower to draw the rest of the information (displayed in pen). For the craft task, we show the collages produced by each participant pair. Names that appear on collages are the intended receivers.

they started to type or were required to provide short answers. CCPs' lengthy explanations to interview questions introduce new topics and information that tighten the relevance constraint and reduced the time ACs have to contribute.

In addition, "guesses" that are phrased as questions meant to anticipate what the $\mathrm{AC}$ is thinking can facilitate information exchange at a lower cost (through a yes/no confirmation) if the CCP's guess is right, but can also reduce ACs' technical agency by limiting their contribution to short answers. These guesses can introduce new ideas that can be hard to repair if they differ from what the AC really wanted to say.

\section{RESULTS: FROM TECHNICAL TO COLLOQUIAL AGENCY}

Our third research question asked: When does technical agency impact colloquial agency? In other words, how does participating in conversation translate to successfully advancing goals? We found that collaboration and agreement between partners is necessary for technical agency to translate into colloquial agency.

In the map task, ACs could express technical agency by selecting a strategy to describe the map route and by contributing detailed directions. Among ACs, only AC4 expressed technical agency by deciding on the strategy:

CCP4: I say divide the paper left and right...

TP: I see the start point on my map, where should I go from there?

AC4: [CCP4], I think you are confusing [experimenter].

AC4: I would go to the g-r-a-v-e-y-a-r-d.

Additionally, AC4 would not only say the landmark's name (as other ACs did) but also followed up to ask where the landmark was located with respect to others. As a result, pair 4 had no inaccurate landmarks, a successful translation of technical agency to colloquial agency.

In pairs other than $\mathrm{P} 4, \mathrm{AC}$ technical and colloquial agency were limited. In these cases, CCPs proposed the strategy used to complete the final map, reducing ACs' technical agency. Their final maps contained inaccurate landmarks, mostly due to misunderstandings of what landmark the $\mathrm{AC}$ was referring to, which was the result of errors in grounding or mutual understanding. These inaccurate landmarks represent a failure of AC colloquial agency, because they were unable to advance their objectives (identifying landmarks) in the task.

\begin{tabular}{lllll}
\hline & P1 & P2 & P3 & P4 \\
\hline Total \# of ideas proposed by AC & 1 & 6 & 4 & 4 \\
Total \# of ideas proposed by CCP & 16 & 16 & 4 & 10 \\
\# collage elements proposed by AC & 1 & 6 & 4 & 4 \\
\# collage elements proposed by CCP with AC agreement & 4 & 4 & 3 & 3 \\
\# collage elements proposed by CCP without AC agreement & 2 & 0 & 1 & 1 \\
\hline
\end{tabular}

Table 3. Collage elements in the final craft that were proposed by the $\mathrm{AC}$, proposed by the $\mathrm{CCP}$ and agreed to by the $\mathrm{AC}$, or proposed by the $\mathrm{CCP}$ and included in the artifact without explicit agreement from the $\mathrm{AC}$.

In contrast, in the craft task, ACs achieved colloquial agency by providing ideas that made it into the craft. All of the ideas proposed by ACs appeared on the final collages, while only $37.5 \%, 25 \%, 80 \%$ and $40 \%$ of ideas proposed by CCP1, CCP2, $\mathrm{CCP} 3$ and CCP4 (respectively) appeared on the final collage. More CCP suggestions did not lead to more CCP ideas on the final craft. In fact, an idea rarely appeared on the final craft without some form of AC input-93\% of CCP ideas on final crafts were first confirmed with AC partners (Table 3). CCP1 and CCP2 asked yes/no questions to confirm all decisions, while CCP3 asked open-ended questions to get ideas from AC3 (e.g., "what is on the beach?"). Pair 4 discussed ideas instead of only confirming CCP4's suggestions:

CCP4: Would you like to do a pineapple? ...

AC4: Thinking-of-a-comma-l-e-i. I was thinking of a lei.

CCP4: A lei, okay. There is pink around somewhere.

[browses crafting materials].

In summary, AC participation impacted the resulting artifacts. The translation from participating ("technical agency") to goal realization ("colloquial agency") was contingent upon cooperation with CCPs, and made possible through interdependent effort, such as having CCPs carry out ideas suggested by ACs.

\section{DISCUSSION AND FUTURE WORK}

Our research confirms agency as a useful frame to consider social interactions of augmented communicators (ACs) in conversation. Constraints of conversation, relationship, task and device create opportunities and challenges for ACs to express agency. Building on our increased understanding of AAC interactions beyond the device, we suggest opportunities for future research to further understand and promote $\mathrm{AC}$ agency under existing constraints. 


\section{Designing for Conversational Constraints}

Conversational constraints are influenced by other speakers, including close conversational partners (CCPs) or unfamiliar third parties (TPs). Our findings suggest that enabling equal control over conversational constraints may lead to more equal participation in conversation.

Participation shift constraint: In the interview task of our study, ACs participated more frequently when directly addressed with an interview question than in subsequent turns. Addressing ACs more frequently in conversation can increase AC participation, and future work could consider how to ensure speakers are addressed equally through technology or conversational methods. For example, prior work aimed at balancing group speaking participation used a rotating robotic microphone that oriented to each speaker in turn [33]. Such work could be extended to balance group communication with ACs by developing social robots that encourage turn taking strategies for speakers with varying speeds (e.g., moving to other speakers while the AC types, and returning to the $\mathrm{AC}$ when they are ready to speak).

Ritual constraint: CCPs increased ACs participation through rituals such as asking ACs permission to answer a question, asking the AC to clarify information, and orienting their bodies towards the AC to ensure their participation. Future work may consider methods and technology that monitor conversation dynamics and leverage rituals to support AC participation when needed. Prior work that monitors dominant speakers and uses gaze of a social robot to guide attention to less dominant speakers [28] could be extended to increase AC agency when necessary. We could also consider methods to teach aspects of CCP rituals to unfamiliar TPs, such as a list of interactive talking points to guide TPs to better support AC agency in conversation.

Relevance Constraint: An AC is disproportionately impacted by the relevance constraint as an $\mathrm{AC}$ has to first type a message before sharing it. Once the AC finishes typing, it might be too late as the topic of the conversation has shifted. Prior work in AAC uses contextual information from the outside world to suggest words and phrases related to the current visual scene [21]. Using additional contextual information from the conversation such as audio, visuals and information on who is speaking could help the AC quickly reply to the conversation topic. Further, an AC could "bookmark" a part of the conversation (e.g., using a short buffered audio recording, or an extracted phrase) to remind other speakers of the original context of a delayed response.

One-speaker constraint: ACs with utterance-level voice output made clear to all speakers that they held the floor and did not experience any missed opportunities caused by erasing a message they wanted to share. Future work may consider other techniques of augmenting ACs' communication with signals that provide insight about ACs' intent to contribute. Prior work that visually displays an AC's status to other speakers (e.g., typing, idle, listening) $[13,32]$ may help decrease the impact of the one-speaker constraint on the $\mathrm{AC}$ by encouraging other speakers to take into account the AC's status. Future work may also consider discrete augmentations that cue partners without distracting their visual attention from the AC, such as wearables or peripheral signals in the environment that are accessible in space and beyond the screen.

Designing for Relationship, Device and Task Constraints Relationship constraint: Similar to Blackstone's "Circles of Communication", our study suggests that ACs communicate differently with different partners [5]. For instance, ACs used nonverbal communication more frequently with their close conversational partner (CCP) than they did with the third party (TP). As CCPs often translated AC nonverbal communication to the third party, future devices could also translate or amplify AC nonverbal communication to other unfamiliar third parties to expand initial communication modalities. We observe beneficial interdependence [3] between the AC-CCP pair, and acknowledging the unique importance of the contributions of each communicative partner can inform new interactions. Considering interdependence, we could extend AAC groupware [13] to consider tools designed specifically for users with different relationships beyond the CCP (e.g., a privacy preserving application for new partners), or for communication groups with many participants.

Device constraint: Device output type (e.g., utterance-level vs phrase-level) impacted ACs' ability to hold the floor once they started speaking. Device input type (e.g., scanning vs direct selection) contributed to the effort it takes for an AC to speak, and prior work suggests that ACs consider whether speaking is "worth the effort" before participating [22]. In the future we should continue to improve device throughput, but also educate non-AC users on how better support AC participation in conversation.

Task constraint: When the CCP knew the information needed to complete a task, the CCP supported the AC through informed participation. When the CCP did not know the information needed for the task, the CCP instead guessed and asked questions to help the pair achieve the goal. Future work could consider how to identify a conversational goal and relevant information from context to better support ACs' expression of agency. For instance, AAC systems could also try to detect and suggest possible general goals of the conversation that could help inform conversational regulation strategies that promote accomplishing this goal.

\section{CONCLUSION}

Our research adds to the body of AAC work in HCI by considering the role of close conversation partners under contextual constraints that, when loosened, create opportunities for augmented communicators to exercise agency. Identifying conversational, task, device and relationship constraints can generate new augmentations that extend beyond the device and to enable different conversational dynamics that favor the expression of agency of augmented communicators.

\section{ACKNOWLEDGEMENTS}

We thank the participants in this study, the CLASS organization, and Patrick Carrington for their invaluable support. 


\section{REFERENCES}

[1] Christine E Ashby. 2011. Whose voice is it anyway?: Giving voice and qualitative research involving individuals that type to communicate. Disability Studies Quarterly 31, 4 (2011).

[2] Salena Babb, Jessica Gormley, David McNaughton, and Janice Light. 2018. Enhancing independent participation within vocational activities for an adolescent with ASD using AAC video visual scene displays. Journal of Special Education Technology (2018), 0162643418795842.

[3] Cynthia L Bennett, Erin Brady, and Stacy M Branham. 2018. Interdependence as a frame for assistive technology research and design. In Proceedings of the 20th International ACM SIGACCESS Conference on Computers and Accessibility. ACM, 161-173.

[4] David R Beukelman and Pat Mirenda. 2013. Augmentative and alternative communication: Supporting children and adults with complex communication needs. Paul H. Brookes Pub.

[5] Sarah W Blackstone. 1999. Circles of communication partners. Augmentative Communication News 12 (1999), $1-2$.

[6] Sarah W. Blackstone and Barbara Collier. 2008. Communication assistants: Human supports for communication access. Augmentative Communication News 20, 3 (2008), 1-15.

[7] Herbert H Clark, Susan E Brennan, and others. 1991. Grounding in communication. Perspectives on socially shared cognition 13, 1991 (1991), 127-149.

[8] Michael Clarke and Andrea Kirton. 2003. Patterns of interaction between children with physical disabilities using augmentative and alternative communication systems and their peers. Child Language Teaching and Therapy 19, 2 (2003), 135-151.

[9] Michael Clarke and Ray Wilkinson. 2008. Interaction between children with cerebral palsy and their peers 2 : Understanding initiated VOCA-mediated turns. Augmentative and Alternative Communication 24, 1 (2008), 3-15.

[10] David Coyle, James Moore, Per Ola Kristensson, Paul Fletcher, and Alan Blackwell. 2012. I did that! Measuring users' experience of agency in their own actions. In Proceedings of the SIGCHI Conference on Human Factors in Computing Systems. ACM, 2025-2034.

[11] Mustafa Emirbayer and Ann Mische. 1998. What is agency? American journal of sociology 103, 4 (1998), 962-1023.

[12] Alexander Fiannaca, Jon Campbell, Ann Paradiso, and Meredith Ringel Morris. 2018. Voicesetting: Voice Authoring UIs for Improved Expressivity in Augmentative Communication. (2018). DOI : http://dx.doi.org/10.1145/3173574.3173857
[13] Alexander Fiannaca, Ann Paradiso, Mira Shah, and Meredith Ringel Morris. 2017. AACrobat: Using mobile devices to lower communication barriers and provide autonomy with gaze-based AAC. In Proceedings of the 2017 ACM Conference on Computer Supported Cooperative Work and Social Computing. ACM, 683-695.

[14] David R Gibson. 2000. Seizing the moment: The problem of conversational agency. Sociological Theory 18, 3 (2000), 368-382.

[15] Erving Goffman and others. 1978. The presentation of self in everyday life. Harmondsworth London.

[16] Charles Goodwin. 2011. Contextures of action. Embodied interaction: Language and body in the material world (2011), 182-193.

[17] Charles Goodwin. 2013. The co-operative, transformative organization of human action and knowledge. Journal of pragmatics 46, 1 (2013), 8-23.

[18] DJ Higginbotham, K Fulcher, and J Seale. 2016. Time and timing in interactions involving individuals with ALS, their unimpaired partners and their speech generating devices. The silent partner (2016), 199-229.

[19] D Jeffery Higginbotham, Ann M Bisantz, Michelle Sunm, Kim Adams, and Fen Yik. 2009. The effect of context priming and task type on augmentative communication performance. Augmentative and Alternative Communication 25, 1 (2009), 19-31.

[20] Seray B Ibrahim, Asimina Vasalou, and Michael Clarke. 2018. Design Opportunities for AAC and Children with Severe Speech and Physical Impairments. In Proceedings of the 2018 CHI Conference on Human Factors in Computing Systems. ACM, 227.

[21] S.K. Kane and M.R. Morris. 2017. Let's Talk about X: Combining image recognition and eye gaze to support conversation for people with ALS. DIS 2017 Proceedings of the 2017 ACM Conference on Designing Interactive Systems (2017), 129-134. DOI: http://dx.doi.org/10.1145/3064663.3064762

[22] Shaun K. Kane, Meredith Ringel Morris, Ann Paradiso, and Jon Campbell. 2017. At Times Avuncular and Cantankerous, with the Reflexes of a Mongoose: Understanding Self-Expression Through Augmentative and Alternative Communication Devices. In Proceedings of the 2017 ACM Conference on Computer Supported Cooperative Work and Social Computing (CSCW'17). ACM, New York, NY, USA, 1166-1179. DOI : http://dx.doi .org/10.1145/2998181.2998284

[23] Janice Light. 1989. Toward a definition of communicative competence for individuals using augmentative and alternative communication systems. Augmentative and Alternative Communication 5, 2 (1989), 137-144. 
[24] Janice Light, Barbara Collier, and Penny Parnes. 1985. Communicative interaction between young nonspeaking physically disabled children and their primary caregivers: Part II-Communicative function. Augmentative and Alternative Communication 1, 3 (1985), 98-107.

[25] Janice Light and David McNaughton. 2014. Communicative competence for individuals who require augmentative and alternative communication: A new definition for a new era of communication? (2014).

[26] Hannah Limerick, David Coyle, and James W Moore. 2014. The experience of agency in human-computer interactions: a review. Frontiers in human neuroscience 8 (2014), 643.

[27] Timothy Mills, H Timothy Bunnell, and Rupal Patel. 2014. Towards personalized speech synthesis for augmentative and alternative communication. Augmentative and Alternative Communication 30, 3 (2014), 226-236.

[28] Yukiko I Nakano, Takashi Yoshino, Misato Yatsushiro, and Yutaka Takase. 2016. Generating robot gaze on the basis of participation roles and dominance estimation in multiparty interaction. ACM Transactions on Interactive Intelligent Systems (TiiS) 5, 4 (2016), 22.

[29] Graham Pullin, Jutta Treviranus, Rupal Patel, and Jeff Higginbotham. 2017. Designing interaction, voice, and inclusion in AAC research. Augmentative and Alternative Communication 33, 3 (2017), 139-148.

[30] Harvey Sacks, Emanuel A Schegloff, and Gail Jefferson. 1978. A simplest systematics for the organization of turn taking for conversation. In Studies in the organization of conversational interaction. Elsevier, 7-55.

[31] Ben Shneiderman. 1986. Eight golden rules of interface design. Disponible en (1986).

[32] Kiley Sobel, Alexander Fiannaca, Jon Campbell, Harish Kulkarni, Ann Paradiso, Ed Cutrell, and Meredith Ringel Morris. 2017. Exploring the Design Space of AAC Awareness Displays. In Proceedings of the 2017 CHI Conference on Human Factors in Computing Systems. ACM, 2890-2903.

[33] Hamish Tennent, Solace Shen, and Malte Jung. 2019. Micbot: A Peripheral Robotic Object to Shape Conversational Dynamics and Team Performance. In 2019 14th ACM/IEEE International Conference on Human-Robot Interaction (HRI). IEEE, 133-142.

[34] Bruce Wisenburn and D Jeffery Higginbotham. 2009. Participant evaluations of rate and communication efficacy of an AAC application using natural language processing. Augmentative and Alternative Communication 25, 2 (2009), 78-89. 